\title{
Power Minimization for Cooperative Wireless Powered Mobile Edge Computing Systems
}

\author{
Xiaoyan $\mathrm{Hu}^{\dagger}$, Kai-Kit Wong ${ }^{\dagger}$, and Kun Yang ${ }^{\ddagger *}$ \\ ${ }^{\dagger}$ Department of Electronic and Electrical Engineering, University College London, London, UK \\ ${ }^{\ddagger}$ School of Computer Science \& Electronic Engineering, University of Essex, Colchester, UK \\ *School of Communication and Information Engineering, UESTC, Chengdu, P. R. China \\ Email: ${ }^{\dagger}\left\{\right.$ xiaoyan.hu.16, kai-kit.wong\}@ucl.ac.uk, ${ }^{\ddagger *}$ kunyang@ $\{$ essex.ac.uk, uestc.edu.cn\}
}

\begin{abstract}
This paper studies the power-efficient joint radio and computational resource allocation for two near-far mobile devices in a wireless powered mobile edge computing system. To overcome the double-near-far effect for the farther device, cooperative communications in the form of relaying via the nearer device is considered for offloading. The AP's total transmit power minimization problem is formulated under the constraints of the computation tasks, which is equivalent to a min-max problem and can be optimally solved by a two-phase method. Numerical results not only shows the significant performance improvement of the proposed scheme, but also demonstrates its effectiveness in handling computation-intensive latency-critical tasks and resisting the double-near-far effect.
\end{abstract}

\section{INTRODUCTION}

With the rapid developments of IoT and $5 \mathrm{G}$ communication technologies, a wide range of emerging mobile applications and mass data processing from mobile social networks [1], [2], have driven the increasing computing demands for mobile devices. In recent years, mobile edge computing (MEC) has emerged as a promising concept, which promotes to use cloudcomputing facilities at the edge of mobile networks, and is motivated by ultralow latency and high bandwidth [3]. The cross-discipline nature of MEC lays the important role of joint radio-and-computational resource management in achieving energy-efficient or delay-optimal MEC [4]-[8]. Nonetheless, insufficient power supply is a major limitation for mobile devices to make full use of powerful resources at the edges. Wireless power transfer (WPT) particularly in the form of wireless powered communication networks (WPCNs) [9] has recently been considered as an important paradigm to provide sustainability for mobile communications. Many works have seen the possible synergy integrating MEC with WPT [4], [8]. However, WPCNs are known to suffer from the "double-nearfar" effect, which occurs because a farther device harvests less energy and is also required to communicate in longer distances [9]. In fact, user cooperation has been extensively investigated in wireless communications to enhance data rate [9]-[11]. Most recently, user cooperation was also considered in MEC [12] to make the most use of the AP's computational resources.

In this paper, we study the wireless powered MEC system to complete the computation-intensive latency-critical (CILC) tasks of two near-far users exploiting cooperative communication. Our objective is to minimize the total transmission power of the AP with joint-optimal power and time allocation [13]. We first formulate AP's transmit power minimization (APTP-
M) problem, and then transform it into an equivalent min-max optimization problem, which is optimally solved by a proposed two-phase method. Simulation results shows that the proposed scheme is very capable of handling CILC tasks and resisting the double-near-far effect in WPCNs.

\section{System Model AND Problem Formulation}

Consider a wireless powered MEC system that consists of a single-antenna AP (with an integrated MEC server), and two single-antenna mobile devices, $D_{i}, i \in\{1,2\}$, both have a CILC task, characterized by $\left\langle I_{i}, C_{i}, T_{i}\right\rangle$, where $I_{i}$ denotes the input data size (in bits), $C_{i}$ is the amount of required computing resource for 1-bit of input data (i.e., the number of CPU cycles required), and $T_{i}$ is the maximum tolerable delay. Assume that the AP has perfect knowledge of the channels and task-related parameters, and it is designed to make resource allocation optimally in a block-based structure where each block has a duration of $T$ seconds. In this paper, we assume that $T_{i}$ for two users is one block length, i.e., $T_{1}=T_{2}=T$.

\section{A. User Cooperation Model for Computation Offloading}

It is assumed that $D_{2}$ is nearer to the AP than $D_{1}$, and we denote the distances between $\mathrm{AP}$ and $D_{1}, \mathrm{AP}$ and $D_{2}$, $D_{1}$ and $D_{2}$ as $d_{1}, d_{2}$, and $d_{12}$, respectively, with $d_{2} \leq d_{1}$. We also assume that $d_{12} \leq d_{1}$ for better cooperation. During the first period $t_{0}$ in a block, AP broadcasts energy in the downlink with power $P_{0}$, and thus the energy harvested by $D_{i}$ is $E_{i}=\nu_{i} g_{i} P_{0} t_{0}$ where $g_{i}$ is the downlink channel power gain from the AP to $D_{i}$ and $0<\nu_{i} \leq 1$ is the corresponding energy conversion efficiency. After the WPT period, $D_{1}$ transmits its input data with power $p_{1}$ during the subsequent period $t_{1}$, and both the AP and $D_{2}$ decode their received signals from $D_{1}$. To overcome the doubly near-far effect, the nearer user $D_{2}$ will then first relay the farther user $D_{1}$ 's information with power $p_{21}$ over period $t_{21}$ and then transmits its own input data to the AP with power $p_{22}$ over period $t_{22}$, all using its harvested energy. We denote the time and power allocation vectors as $\mathbf{t}=\left[t_{0}, t_{1}, t_{21}, t_{22}\right]$ and $\mathbf{p}=\left[p_{1}, p_{21}, p_{22}\right]$, respectively. According to the results of [9], [11], the offloaded data size of $D_{1}$ for computation at the AP can be expressed as

$$
L_{1}(\mathbf{t}, \mathbf{p})=\min \left\{L_{1,1}(\mathbf{t}, \mathbf{p})+L_{1,2}(\mathbf{t}, \mathbf{p}), L_{1,12}(\mathbf{t}, \mathbf{p})\right\},
$$

where $L_{1,1}(\mathbf{t}, \mathbf{p}), L_{1,2}(\mathbf{t}, \mathbf{p})$ and $L_{1,12}(\mathbf{t}, \mathbf{p})$ denote $D_{1}$ 's offloaded data size from $D_{1}$ to the AP, from $D_{2}$ to the AP, and 
from $D_{1}$ to $D_{2}$, respectively, which are given by

$$
\begin{aligned}
L_{1,1}(\mathbf{t}, \mathbf{p}) & =t_{1} r_{1,1}(\mathbf{p})=t_{1} B \log _{2}\left(1+p_{1} h_{1} / N_{0}\right) \\
L_{1,2}(\mathbf{t}, \mathbf{p}) & =t_{21} r_{1,2}(\mathbf{p})=t_{21} B \log _{2}\left(1+p_{21} h_{2} / N_{0}\right), \\
L_{1,12}(\mathbf{t}, \mathbf{p}) & =t_{1} r_{1,12}(\mathbf{p})=t_{1} B \log _{2}\left(1+p_{1} h_{12} / N_{0}\right)
\end{aligned}
$$

where $r_{1,1}(\mathbf{p}), r_{1,2}(\mathbf{p})$, and $r_{1,12}(\mathbf{p})$ are the corresponding transmission rates, and $h_{1}, h_{2}$ and $h_{12}$ are the channel power gains from $D_{1}, D_{2}$ to the AP, and from $D_{1}$ to $D_{2}$, respectively. ${ }^{1}$ Also, $B$ is the channel bandwidth, and $N_{0}$ is the receiver noise power at the AP and $D_{2}$. Similarly, the offloaded data size of $D_{2}$ for computing at the AP is described as

$$
L_{2}(\mathbf{t}, \mathbf{p})=t_{22} r_{2}(\mathbf{p})=t_{22} B \log _{2}\left(1+p_{22} h_{2} / N_{0}\right) .
$$

According to the task model, the constraint $L_{i}(\mathbf{t}, \mathbf{p}) \leq I_{i}, i \in$ $\{1,2\}$ should be satisfied. In this paper, we mainly consider the WPT time and the offloading time as the total latency of the WPT-MEC system, and thus we obtain a latency constraint given by $t_{0}+t_{1}+t_{21}+t_{22} \leq T$. Besides, the energy consumption of $D_{1}$ and $D_{2}$ for computation offloading equals to the energy consumed for wireless transmissions, given by

$$
E_{\text {off }, 1}(\mathbf{t}, \mathbf{p})=p_{1} t_{1}, \quad E_{\text {off }, 2}(\mathbf{t}, \mathbf{p})=p_{21} t_{21}+p_{22} t_{22} .
$$

\section{B. Local Computing Model}

Given a pair of $(\mathbf{t}, \mathbf{p})$, the offloaded data sizes $\left\{L_{i}(\mathbf{t}, \mathbf{p})\right\}$ will be known, and hence the remaining input data of each user, i.e., $I_{i}-L_{i}(\mathbf{t}, \mathbf{p})$, should be computed locally at $D_{i}$, $i \in\{1,2\}$. For local computing, we assume that the CPU frequency is fixed as $f_{i}$ for $D_{i}$. In order to satisfy the latency constraint, i.e., $\left(I_{i}-L_{i}(\mathbf{t}, \mathbf{p})\right) C_{i} / f_{i} \leq T$, the offloaded data for $D_{i}$ should have a minimum size of $L_{i}(\mathbf{t}, \mathbf{p}) \geq$ $M_{i}^{+}=\max \left\{I_{i}-f_{i} T / C_{i}, 0\right\}$. The energy consumption per CPU cycle is denoted as $Q_{i}=\kappa_{i} f_{i}^{2}$, where $\kappa_{i}$ is the effective capacitance coefficient, and thus the energy consumption of $D_{i}$ for local computing can be expressed as

$$
E_{\mathrm{loc}, i}(\mathbf{t}, \mathbf{p})=\left(I_{i}-L_{i}(\mathbf{t}, \mathbf{p})\right) C_{i} Q_{i}, \quad i \in\{1,2\} .
$$

\section{Problem Formulation}

Based on the model, the energy saving for $D_{i}, i \in\{1,2\}$ is

$$
E_{\mathrm{s}, i}\left(P_{0}, \mathbf{t}, \mathbf{p}\right)=\nu_{i} g_{i} P_{0} t_{0}-E_{\mathrm{off}, i}(\mathbf{t}, \mathbf{p})-E_{\mathrm{loc}, i}(\mathbf{t}, \mathbf{p}) .
$$

Furthermore, the APTPM problem for minimizing AP's transmit power can be formulated as problem (P1) below

$$
\begin{array}{cl}
\underset{P_{0}>0, \mathbf{t}, \mathbf{p}}{\min } & P_{0} \\
\text { s.t. } & E_{\mathrm{s}, i}\left(P_{0}, \mathbf{t}, \mathbf{p}\right) \geq 0, \quad i \in\{1,2\}, \\
& M_{i}^{+} \leq L_{i}(\mathbf{t}, \mathbf{p}) \leq I_{i}, \quad i \in\{1,2\}, \\
& T-\left(t_{0}+t_{1}+t_{21}+t_{22}\right) \geq 0, \\
& t_{0} \geq 0, t_{1} \geq 0, t_{21} \geq 0, t_{22} \geq 0, \\
& p_{1} \geq 0, p_{21} \geq 0, p_{22} \geq 0 .
\end{array}
$$

Note that problem (P1) is nonconvex because of the nonconvex expressions of $L_{i}(\mathbf{t}, \mathbf{p})$, and the product of $P_{0} t_{0}$ in $E_{\mathrm{s}, i}\left(P_{0}, \mathbf{t}, \mathbf{p}\right), i \in\{1,2\}$. In the sequel, we will focus on removing these two obstacles to make problem (P1) solvable.

\footnotetext{
${ }^{1}$ In order to investigate the effect of user cooperation in resisting the doublenear-far problem, we mainly consider the case of $h_{1}<h_{12}$.
}

\section{Efforts to Make Problem (P1) Solvable}

\section{A. Transforming $L_{1}(\mathbf{t}, \mathbf{p})$ and $L_{2}(\mathbf{t}, \mathbf{p})$ into Convex}

By introducing the variables $q_{1}=\frac{p_{1} t_{1}}{\nu_{1} g_{1} P_{0}}, q_{21}=\frac{p_{21} t_{21}}{\nu_{2} g_{2} P_{0}}$ and denoting $\mathbf{q}=\left[q_{1}, q_{21}\right], L_{1,1}(\mathbf{t}, \mathbf{p}), L_{1,2}(\mathbf{t}, \mathbf{p})$ and $L_{1,12}(\mathbf{t}, \mathbf{p})$ in (2)-(4) can then be re-expressed as functions of $\mathbf{t}$ and $\mathbf{q}$ as

$$
\begin{aligned}
L_{1,1}(\mathbf{t}, \mathbf{q}) & =t_{1} B \log _{2}\left(1+\beta_{1} P_{0} q_{1} / t_{1}\right), \\
L_{1,2}(\mathbf{t}, \mathbf{q}) & =t_{21} B \log _{2}\left(1+\beta_{2} P_{0} q_{21} / t_{21}\right), \\
L_{1,12}(\mathbf{t}, \mathbf{q}) & =t_{1} B \log _{2}\left(1+\beta_{12} P_{0} q_{1} / t_{1}\right),
\end{aligned}
$$

where $\beta_{1}=\frac{\nu_{1} g_{1} h_{1}}{N_{0}}, \beta_{2}=\frac{\nu_{2} g_{2} h_{2}}{N_{0}}$, and $\beta_{12}=\frac{\nu_{1} g_{1} h_{12}}{N_{0}}$. Note that the above three functions equal to 0 when $t_{1}=0, t_{21}=0$ and $t_{1}=0$, respectively. Using the property of perspective function [14], it is easily verified that $L_{1,1}(\mathbf{t}, \mathbf{q}), L_{1,2}(\mathbf{t}, \mathbf{q})$ and $L_{1,12}(\mathbf{t}, \mathbf{q})$ are all joint concave functions of $\mathbf{t}$ and $\mathbf{q}$. Next, we introduce a new variable

$$
L_{1}=\min \left\{L_{1,1}(\mathbf{t}, \mathbf{q})+L_{1,2}(\mathbf{t}, \mathbf{q}), L_{1,12}(\mathbf{t}, \mathbf{q})\right\}
$$

to replace $L_{1}(\mathbf{t}, \mathbf{p})$ in (P1) with two additional convex constraints, $L_{1,1}(\mathbf{t}, \mathbf{q})+L_{1,2}(\mathbf{t}, \mathbf{q}) \geq L_{1}$ and $L_{1,12}(\mathbf{t}, \mathbf{q}) \geq L_{1}$. Besides, we redefine the offloaded data size of $D_{2}$ as an independent variable $L_{2}$, and then by defining a function $g(x)=N_{0}\left(2^{\frac{x}{B}}-1\right), x \geq 0$, the offloading power $p_{22}$ can be described as $p_{22}=\frac{1}{h_{2}} g\left(\frac{L_{2}}{t_{22}}\right)$ according to (5). Hence, the energy savings for $D_{1}$ and $D_{2}$ can be rewritten as

$$
\begin{aligned}
E_{\mathrm{s}, 1}\left(P_{0}, \mathbf{t}, \mathbf{q}, L_{1}\right)= & \nu_{1} g_{1} P_{0}\left(t_{0}-q_{1}\right)-\left(I_{1}-L_{1}\right) G_{1}, \\
E_{\mathrm{s}, 2}\left(P_{0}, \mathbf{t}, \mathbf{q}, L_{2}\right)= & \nu_{2} g_{2} P_{0}\left(t_{0}-q_{21}\right) \\
& -\frac{t_{22}}{h_{2}} g\left(\frac{L_{2}}{t_{22}}\right)-\left(I_{2}-L_{2}\right) G_{2},
\end{aligned}
$$

where $G_{i}=C_{i} Q_{i}, i \in\{1,2\}$. As $g(x)$ is a convex function, its perspective function $t_{22} g\left(\frac{L_{2}}{t_{22}}\right)$ is a joint convex function of $t_{22}$ and $L_{2}$ considering both the cases of $t_{22}>0$ and $t_{22}=$ 0 [14]. Hence, problem (P1) can be equivalent transformed into another APTPM problem (P2),

$$
\begin{array}{cl}
\min _{P_{0}>0, \mathbf{t}, \mathbf{q}, L_{1}, L_{2}} & P_{0} \\
\text { s.t. } & E_{\mathrm{s}, i}\left(P_{0}, \mathbf{t}, \mathbf{q}, L_{i}\right) \geq 0, i \in\{1,2\}, \\
& L_{1,1}(\mathbf{t}, \mathbf{q})+L_{1,2}(\mathbf{t}, \mathbf{q}) \geq L_{1}, \\
& L_{1,12}(\mathbf{t}, \mathbf{q}) \geq L_{1}, \\
& M_{i}^{+} \leq L_{i} \leq I_{i}, i \in\{1,2\}, \\
& T-\left(t_{0}+t_{1}+t_{21}+t_{22}\right) \geq 0, \\
& t_{0} \geq 0, t_{1} \geq 0, t_{21} \geq 0, t_{22} \geq 0, \\
& q_{1} \geq 0, q_{21} \geq 0 .
\end{array}
$$

In problem (P2), only constraints in (16b) remain non-convex since $P_{0}$ is coupled with $t_{0}, q_{1}$ and $q_{21}$. Next, we will separate this coupling to facilitate the problem solving.

\section{B. Separation for the Coupled Parameters}

In order to further solve the APTPM problem (P2), we first introduce an equivalent min-max optimization problem (P3):

$$
\begin{array}{ll}
\min _{P_{0}>0} \max _{\substack{\mathbf{t}, \mathbf{q}, L_{1}, L_{2} \\
\text { s.t. }}} & \sum_{i=1}^{2} E_{\mathrm{s}, i}\left(P_{0}, \mathbf{t}, \mathbf{q}, L_{i}\right) \\
\text { (16b)-(16h), } &
\end{array}
$$


which is still nonconvex in the above form, but can be optimally solved by a two-phase method. In the first phase, we solve the inner sub-problem with a given $P_{0}$ where the sum-energy-saving (SES) is maximized under the constraints in (P3), referred to as the SESM problem (P4):

$$
\begin{array}{cl}
\max _{\mathbf{t}, \mathbf{q}, L_{1}, L_{2}} & E_{\mathrm{s}, 1}\left(\mathbf{t}, \mathbf{q}, L_{1}\right)+E_{\mathrm{s}, 2}\left(\mathbf{t}, \mathbf{q}, L_{2}\right) \\
\text { s.t. } & (16 \mathrm{~b})-(16 \mathrm{~h}),
\end{array}
$$

where is a convex optimization problem, through which the optimal $\mathbf{t}^{*}, \mathbf{q}^{*}$ (or $\mathbf{p}^{*}$ ), $L_{1}^{*}$ and $L_{2}^{*}$ corresponding to the given $P_{0}$ can be obtained. It is easy to understand that if we assume the given $P_{0}$ is the optimal minimum, then the obtained $\left(\mathbf{t}^{*}, \mathbf{p}^{*}, L_{1}^{*}, L_{2}^{*}\right)$ is actually the optimal solution to problem (P2). If we find the minimum given $P_{0}^{\star}$ that maximizes the SES, then the obtained $\left(P_{0}^{\star}, \mathbf{t}^{\star}, \mathbf{p}^{\star}, L_{1}^{\star}, L_{2}^{\star}\right)$, i.e., the optimal solution of (P3), is actually the joint-optimal solution of (P2). In the second phase of the method, we will find the minimum $P_{0}$ by a bi-section search method. In the following section, we will demonstrate the problem-solving process of problem (P3) with the proposed two-phase method.

\section{The Two-Phase Method for Cooperative MeC}

\section{A. Problem-Solving with Lagrange Method}

To gain more insights of the solution to problem (P4), we next solve it optimally using the Lagrange method [14]. The partial Lagrange function of (P4) is defined as

$$
\begin{aligned}
& \mathcal{L}\left(\mathbf{t}, \mathbf{q}, L_{1}, L_{2}, \eta, \boldsymbol{\lambda}\right) \\
\triangleq & \left(1+\lambda_{1}\right) E_{\mathrm{s}, 1}\left(\mathbf{t}, \mathbf{q}, L_{1}\right)+\left(1+\lambda_{2}\right) E_{\mathrm{s}, 2}\left(\mathbf{t}, \mathbf{q}, L_{2}\right) \\
& +\lambda_{3}\left(L_{1,1}(\mathbf{t}, \mathbf{q})+L_{1,2}(\mathbf{t}, \mathbf{q})-L_{1}\right) \\
& +\lambda_{4}\left(L_{1,12}(\mathbf{t}, \mathbf{q})-L_{1}\right) \\
& +\eta\left(T-\left(t_{0}+t_{1}+t_{21}+t_{22}\right)\right),
\end{aligned}
$$

where $\boldsymbol{\lambda}=\left[\lambda_{1}, \ldots, \lambda_{4}\right] \succeq \mathbf{0}$ ( $\succeq$ denotes the componentwise inequality) and $\eta \geq 0$ consist of the Lagrange multipliers associated with the constraints (16b)-(16d) and (16f) in problem (P4), respectively. In order to facilitate the analysis in the sequel, we define another two functions

$$
f(x)=\ln (1+x)+\frac{1}{1+x}-1, h(x)=g(x)-x g^{\prime}(x) .
$$

Hence, the following lemma is established, which can be easily proved using the property of Lambert function.

Lemma 1. $f(x)(g(x))$ is a monotonic increasing (decreasing) function of $x \geq 0$ with $f(0)=0(g(0)=$ $0)$. Given $C>0(G<0)$, there exists a unique positive solution for equation $f(x)=C(g(x)=G)$, given by $x^{*}=-\left(1+\left(W_{0}\left(-e^{(-(C+1))}\right)\right)^{-1}\right) \quad\left(x^{*}=\right.$ $\left.\frac{B}{\ln 2}\left[W_{0}\left(\frac{G / N_{0}+1}{-e}\right)+1\right]\right)$, where $W_{0}(z)$ is the principal branch of the lambert $W$ function [15], and $e$ is the base of the natural logarithm.

We first assume that (P4) is feasible and let $\lambda^{*}, \eta^{*}$ denote the optimal Lagrange multipliers under a given $P_{0}$. Then applying the Karush-Kuhn-Tucker (KKT) conditions [14] leads to the following necessary and sufficient conditions:

$$
\begin{aligned}
& \frac{\partial \mathcal{L}}{\partial t_{0}^{*}}=\left(1+\lambda_{1}^{*}\right) \nu_{1} g_{1} P_{0}+\left(1+\lambda_{2}^{*}\right) \nu_{2} g_{2} P_{0}-\eta^{*}=0, \\
& \frac{\partial \mathcal{L}}{\partial t_{1}^{*}}=\frac{B \lambda_{3}^{*}}{\ln 2} f\left(\beta_{1} P_{0} \frac{q_{1}^{*}}{t_{1}^{*}}\right)+\frac{B \lambda_{4}^{*}}{\ln 2} f\left(\beta_{12} P_{0} \frac{q_{1}^{*}}{t_{1}^{*}}\right)=\eta^{*}, \\
& \frac{\partial \mathcal{L}}{\partial t_{21}^{*}}=\frac{B \lambda_{3}^{*}}{\ln 2} f\left(\beta_{2} P_{0} \frac{q_{21}^{*}}{t_{21}^{*}}\right)-\eta^{*}=0, \\
& \frac{\partial \mathcal{L}}{\partial t_{22}^{*}}=-\left(1+\lambda_{2}^{*}\right) \frac{1}{h_{2}} h\left(\frac{L_{2}^{*}}{t_{22}^{*}}\right)-\eta^{*}=0, \\
& \frac{\partial \mathcal{L}}{\partial q_{1}^{*}}=-\left(1+\lambda_{1}^{*}\right) \nu_{1} g_{1} P_{0}+\frac{B}{\ln 2} F\left(t_{1}^{*}, q_{1}^{*}, \lambda_{3}^{*}, \lambda_{4}^{*}\right)=0, \\
& \frac{\partial \mathcal{L}}{\partial q_{21}^{*}}=\frac{B}{\ln 2}\left(\frac{\lambda_{3}^{*} \beta_{2} P_{0}}{1+\beta_{2} P_{0}}\right)-\left(1+\lambda_{21}^{*}\right) \nu_{2} g_{2} P_{0}=0, \\
& \frac{\partial \mathcal{L}}{\partial L_{1}^{*}}=\left(1+\lambda_{1}^{*}\right) G_{1}-\lambda_{3}^{*}-\lambda_{4}^{*}\left\{\begin{array}{l}
<0, L_{1}^{*}=M_{1}^{+}, \\
=0, L_{1}^{*} \in\left(M_{1}^{+}, I_{1}\right), \\
>0, L_{1}^{*}=I_{1},
\end{array}\right. \\
& \frac{\partial \mathcal{L}}{\partial L_{2}^{*}}=\left(1+\lambda_{2}^{*}\right) H\left(\frac{L_{2}^{*}}{t_{22}^{*}}\right)\left\{\begin{array}{l}
<0, L_{2}^{*}=M_{2}^{+}, \\
=0, L_{2}^{*} \in\left(M_{2}^{+}, I_{2}\right), \\
>0, L_{2}^{*}=I_{2},
\end{array}\right. \\
& \lambda_{1}^{*} E_{\mathrm{s}, 1}\left(\mathbf{t}^{*}, \mathbf{q}^{*}, L_{1}^{*}\right)=0, \lambda_{2}^{*} E_{\mathrm{s}, 2}\left(\mathbf{t}^{*}, \mathbf{q}^{*}, L_{2}^{*}\right)=0 \\
& \lambda_{3}^{*}\left(L_{1,1}\left(\mathbf{t}^{*}, \mathbf{q}^{*}\right)+L_{1,2}\left(\mathbf{t}^{*}, \mathbf{q}^{*}\right)-L_{1}^{*}\right)=0, \\
& \lambda_{4}^{*}\left(L_{1,12}\left(\mathbf{t}^{*}, \mathbf{q}^{*}\right)-L_{1}^{*}\right)=0, \\
& \eta^{*}\left(T-\left(t_{0}^{*}+t_{1}^{*}+t_{21}^{*}+t_{22}^{*}\right)\right)=0 .
\end{aligned}
$$

where $F\left(t_{1}^{*}, q_{1}^{*}, \lambda_{3}^{*}, \lambda_{4}^{*}\right)=\frac{\lambda_{3}^{*} \beta_{1} P_{0}}{1+\beta_{1} P_{0} \frac{q_{1}^{*}}{t_{1}^{*}}}+\frac{\lambda_{4}^{*} \beta_{12} P_{0}}{1+\beta_{12} P_{0} \frac{q_{1}^{*}}{t_{1}^{*}}}, H\left(\frac{L_{2}^{*}}{t_{22}^{*}}\right)=$ $G_{2}-\frac{1}{h_{2}} g^{\prime}\left(\frac{L_{2}^{*}}{t_{22}^{*}}\right)$. Note that $t_{0}^{*}+t_{1}^{*}+t_{21}^{*}+t_{22}^{*}=T$ must hold; otherwise, we can always allocate the remaining time to $t_{0}^{*}$ to further increase the energy saving of both two users, and thus $\eta^{*}>0$ holds for sure. Furthermore, the following lemma describes an important result concerning $\mathbf{t}^{*}, \mathbf{q}^{*}$ and $L_{1}^{*}$.

Lemma 2. The optimal time and power allocation $\left(\mathbf{t}^{*}, \mathbf{q}^{*}\right)$ ensures the following property of $D_{1}$ 's offloaded data size, $L_{1}^{*}$.

$$
L_{1}^{*}=L_{1,1}\left(\mathbf{t}^{*}, \mathbf{q}^{*}\right)+L_{1,2}\left(\mathbf{t}^{*}, \mathbf{q}^{*}\right) \leq L_{1,12}\left(\mathbf{t}^{*}, \mathbf{q}^{*}\right) .
$$

Proof. We know that $\frac{\partial\left(t_{22} g\left(\frac{L_{2}}{t_{22}}\right)\right)}{\partial t_{22}}=h\left(\frac{L_{2}}{t_{22}}\right)<0$ for $t_{22}>0$, which indicates that $t_{22} g\left(\frac{L_{2}}{t_{22}}\right)$ is a monotonically decreasing function of $t_{22}$. It is easy to prove that the inequality $L_{1,1}\left(\mathbf{t}^{*}, \mathbf{q}^{*}\right)<L_{1,12}\left(\mathbf{t}^{*}, \mathbf{q}^{*}\right)$ always holds for the considered case of $h_{1}<h_{12}$. If $L_{1,1}\left(\mathbf{t}^{*}, \mathbf{q}^{*}\right)+L_{1,2}\left(\mathbf{t}^{*}, \mathbf{q}^{*}\right)>$ $L_{1,12}\left(\mathbf{t}^{*}, \mathbf{q}^{*}\right)$ holds, we can always allocate part of $t_{21}^{*}$ to $t_{22}^{*}$ while maintaining the same $L_{1}^{*}, L_{2}^{*}, \mathbf{q}^{*}, t_{0}^{*}, t_{1}^{*}$ and the sum of $t_{21}^{*}, t_{22}^{*}$, which will decrease $L_{1,2}\left(\mathbf{t}^{*}, \mathbf{q}^{*}\right)$ until the equality holds. This operation will result in an increased $E_{\mathrm{S}, 2}\left(\mathbf{t}^{*}, \mathbf{q}^{*}, L_{2}^{*}\right)$ by decreasing $t_{22}^{*} g\left(\frac{L_{2}^{*}}{t_{22}^{*}}\right)$ without reducing $E_{\mathrm{s}, 1}\left(\mathbf{t}^{*}, \mathbf{q}^{*}, L_{1}^{*}\right)$, and thus will increase the objective function of problem (P4). Hence, expression (33) always holds with the optimal solution of problem (P4). 
Based on the result of Lemma 2, we can derive that $\lambda_{3}^{*}>0$ and $\lambda_{4}^{*}=0$. Furthermore, for $\mathbf{t}^{*} \succ \mathbf{0}$ and $\mathbf{q}^{*} \succ \mathbf{0}$, it can be derived from (22), (23) and Lemma 1 that

$$
\beta_{1} \frac{q_{1}^{*}}{t_{1}^{*}}=\beta_{2} \frac{q_{21}^{*}}{t_{21}^{*}}=-\frac{1}{P_{0}}\left(1+\left(W_{0}\left(-e^{-\left(\frac{\eta^{*} \ln 2}{\lambda_{3}^{*} B}+1\right)}\right)\right)^{-1}\right) .
$$

Moreover, through the KKT conditions (25) and (26), we can respectively derive that

$$
\begin{aligned}
\beta_{1} \frac{q_{1}^{*}}{t_{1}^{*}} & =\frac{\lambda_{3}^{*} B \beta_{1}}{\left(1+\lambda_{1}^{*}\right) \nu_{1} g_{1} P_{0} \ln 2}-\frac{1}{P_{0}}, \\
\beta_{2} \frac{q_{21}^{*}}{t_{21}^{*}} & =\frac{\lambda_{3}^{*} B \beta_{2}}{\left(1+\lambda_{2}^{*}\right) \nu_{2} g_{2} P_{0} \ln 2}-\frac{1}{P_{0}} .
\end{aligned}
$$

Based on (34)-(36), we obtain that $\left(1+\lambda_{1}^{*}\right) \nu_{1} g_{1} P_{0}=$ $\frac{\beta_{1}}{\beta_{2}}\left(1+\lambda_{2}^{*}\right) \nu_{2} g_{2} P_{0}$. Combining the condition (21), the optimal Lagrange multipliers have the following property:

$$
\left(1+\lambda_{i}^{*}\right) \nu_{i} g_{i} P_{0}=\frac{\beta_{i} \eta^{*}}{\beta_{1}+\beta_{2}}, i \in\{1,2\} .
$$

Hence, by substituting (37) into (35) and (36), we obtain

$$
\beta_{1} \frac{q_{1}^{*}}{t_{1}^{*}}=\beta_{2} \frac{q_{21}^{*}}{t_{21}^{*}}=\frac{B \lambda_{3}^{*}\left(\beta_{1}+\beta_{2}\right)}{\eta^{*} \ln 2}-\frac{1}{P_{0}} .
$$

Based on these results, the optimal resource allocation of (P4) for a given $P_{0}$ is characterized in the following subsections.

\section{B. Optimal Offloading Decisions with Power Allocation}

First, we define an offloading indicator for $D_{i}$ as $\mu_{i} \triangleq$ $B h_{i} C_{i} Q_{i} /\left(N_{0} \ln 2\right), i \in\{1,2\}$. Note that $\mu_{i}$ depends on the variables quantifying uplink channel $\left(h_{i}\right)$, local computing $\left(C_{i} Q_{i}\right)$, and it is a monotonically increasing function of $h_{i}, C_{i}$ and $Q_{i}$. The following theorem shows the relationship between the optimal solution with $\mu_{i}$.

Theorem 1. (Optimal Cooperative Computation Offloading Decisions with Power Allocation).

1) If $M_{1}^{+}>0$ or $\mu_{1} \geq\left(\beta_{1}+\beta_{2}\right) P_{0} / z^{*}$, the optimal $L_{1}^{*}, p_{1}^{*}$ and $p_{21}^{*}$ (all in semi-closed from) can be expressed as

$$
\begin{aligned}
& L_{1}^{*} \begin{cases}=M_{1}^{+}, & \mu_{1}<\left(\beta_{1}+\beta_{2}\right) P_{0} / z^{*}, \\
\in\left(M_{1}^{+}, I_{1}\right), & \mu_{1}=\left(\beta_{1}+\beta_{2}\right) P_{0} / z^{*}, \\
=I_{1}, & \mu_{1}>\left(\beta_{1}+\beta_{2}\right) P_{0} / z^{*},\end{cases} \\
& p_{1}^{*}=\frac{N_{0}}{h_{1}}\left(\left(\beta_{1}+\beta_{2}\right) P_{0} / z^{*}-1\right)>0, \\
& p_{21}^{*}=\frac{N_{0}}{h_{2}}\left(\left(\beta_{1}+\beta_{2}\right) P_{0} / z^{*}-1\right)>0,
\end{aligned}
$$

in which $z^{*}$ is the unique solution of the equation given by $e^{\left(\frac{1}{\left(\beta_{1}+\beta_{2}\right) P_{0}}-1\right) z}-\frac{e}{\left(\beta_{1}+\beta_{2}\right) P_{0}} z=0$ on the specific range of $z \in\left(0,\left(\beta_{1}+\beta_{2}\right) P_{0}\right)$. If $M_{1}^{+}=0$ and $\mu_{1}<\left(\beta_{1}+\beta_{2}\right) P_{0} / z^{*}$, it is optimal to set $L_{1}^{*}=0, p_{1}^{*}=0$, and $p_{21}^{*}=0$.

2) If $M_{2}^{+}>0$ or $\rho\left(\mu_{2}\right) \geq\left(\beta_{1}+\beta_{2}\right) P_{0}$, the optimal $L_{2}^{*}$ and $p_{22}^{*}$ (all in closed form) are given by

$$
\begin{aligned}
& \left.\left.L_{2}^{*}\left\{\begin{array}{ll}
=M_{2}^{+}, & \rho\left(\mu_{2}\right)<\left(\beta_{1}+\beta_{2}\right) P_{0}, \\
\in\left(M_{2}^{+}, I_{2}\right), & \rho\left(\mu_{2}\right)=\left(\beta_{1}+\beta_{2}\right) P_{0}, \\
=I_{2}, & \rho\left(\mu_{2}\right)>\left(\beta_{1}+\beta_{2}\right) P_{0},
\end{array}\right)+1\right]\right), \\
& p_{22}^{*}=\frac{1}{h_{2}} g\left(\frac { B } { \operatorname { l n } 2 } \left[W_{0}\left(\frac{\left(\beta_{1}+\beta_{2}\right) P_{0}-1}{e}\right)+1\right.\right.
\end{aligned}
$$

where $\rho\left(\mu_{2}\right) \triangleq \mu_{2} \ln \mu_{2}-\mu_{2}+1$. If $M_{2}^{+}=0$ and $\rho\left(\mu_{2}\right)<$ $\left(\beta_{1}+\beta_{2}\right) P_{0}$, it is optimal to set $L_{2}^{*}=0$ and $p_{22}^{*}=0$.

Proof. See Appendix A.

Theorem 1 shows that $L_{i}, i \in\{1,2\}$ have a thresholdbased structure. Since the exact cases of $\mu_{1}=\left(\beta_{1}+\beta_{2}\right) P_{0} / z^{*}$ in (39) and $\rho\left(\mu_{2}\right)=\left(\beta_{1}+\beta_{2}\right) P_{0}$ in (42) rarely occur in practice, the optimal policy makes binary offloading decisions for both cooperative users. Besides, $L_{i}$ grows with increasing $\mu_{i}$, which is consistent with the intuition that more resources should be scheduled to computation offloading when users have good channels (i.e., large $h_{i}$ ) or consume high local computing energy (i.e., large $C_{i}$ and $Q_{i}$ ). Moreover, the same item in the thresholds of the offloading decisions for two users, i.e., $\left(\beta_{1}+\beta_{2}\right)=\left(\nu_{1} g_{1} h_{1}+\nu_{2} g_{2} h_{2}\right) / N_{0}$ reflects the energy harvesting potential of two users (i.e., $\nu_{1} g_{1}$ and $\nu_{2} g_{2}$ ) and the quality of uplink offloading channels (i.e., $h_{1}$ and $h_{2}$ ), which demonstrates the effect of user cooperation that either user's offloading decision is affected by the other user's energyharvesting ability and offloading-channel quality. Based on the results in Theorem 1, it is easy to obtain the following lemma.

Lemma 3. For $L_{1}^{*}>0$, the optimal transmit rates of $D_{1}$ and $D_{2}$ for offloading $D_{1}$ 's input data are same, i.e.,

$$
r_{1,1}\left(\mathbf{p}^{*}\right)=r_{1,2}\left(\mathbf{p}^{*}\right)=B \log _{2}\left(\left(\beta_{1}+\beta_{2}\right) P_{0} / z^{*}\right) .
$$

\section{Optimal Power-Efficient Time Allocation}

The corresponding optimal time allocation, i.e., $\mathbf{t}^{*}=$ $\left(t_{0}^{*}, t_{1}^{*}, t_{21}^{*}, t_{22}^{*}\right)$, is summarised in Theorem 2 .

Theorem 2. (Optimal Time Allocation for WPT and Cooperative Computation Offloading).

1) The optimal time allocation for offloading $D_{2}$ 's input data is given by

$$
t_{22}^{*}=\frac{L_{2}^{*} \ln 2}{B}\left[W_{0}\left(\frac{\left(\beta_{1}+\beta_{2}\right) P_{0}-1}{e}\right)+1\right]^{-1} .
$$

2) The optimal WPT duration time can be derived as

$$
t_{0}^{*}=T-t_{22}^{*}-L_{1}^{*} / r_{1,1}\left(\mathbf{p}^{*}\right) .
$$

3) The optimal time allocation for offloading $D_{1}$ 's input data, i.e., $\left(t_{1}^{*}, t_{21}^{*}\right)$ can be expressed $a s^{2}$

$$
\left(t_{1}^{*}, t_{21}^{*}\right)=\left(L_{1}^{*} / r_{1,12}\left(\mathbf{p}^{*}\right), L_{1}^{*} / r_{1,1}\left(\mathbf{p}^{*}\right)-t_{1}^{*}\right),
$$

where $\left(t_{1}^{*}, t_{21}^{*}\right)=(0,0)$ when $L_{1}^{*}=0$.

Proof. See Appendix B.

\section{Optimal Resource Allocation for obtaining $P_{0}^{\star}$}

In this section, we will discuss the second phase of solving problem (P2). Note that with a larger $P_{0}$, the feasible region of problem (P4) will be lager as well, and thus more energy will be saved, which means that the maximum SES obtained by (P4) is an increasing function of $P_{0}$. Hence, the minimum $P_{0}^{\star}$ of the original APTPM problem (P1) can be obtained through

\footnotetext{
${ }^{2}$ In this paper, we mainly consider the usual encountered case of $h_{1}<h_{2}$. Actually, if the case of $h_{1}>h_{2}$ does happen, we can simply exchange the roles of the two devices to apply the proposed scheme.
} 

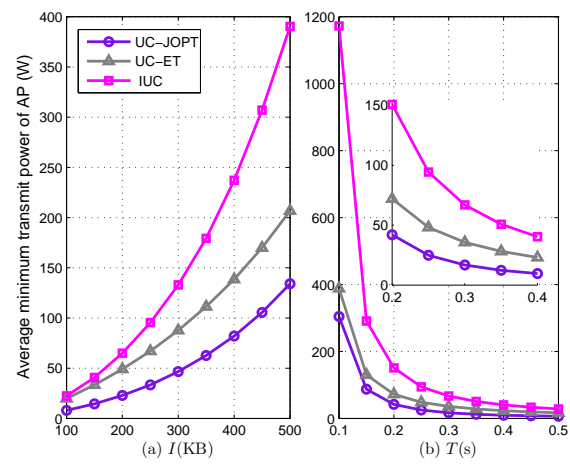

Fig. 1. Average minimum transmit power of AP versus $I$ and $T$.

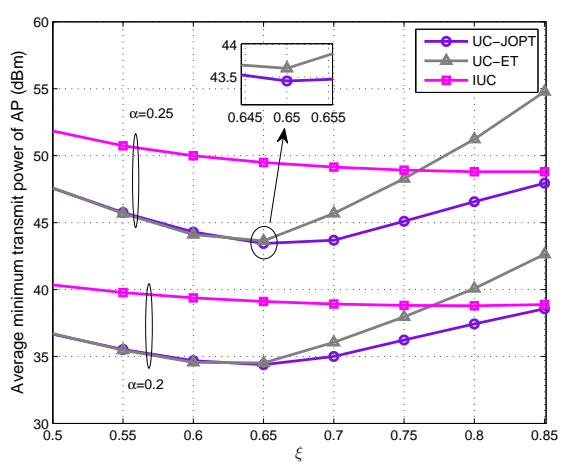

Fig. 2. Average minimum transmit power of AP versus $\xi$. a bisection search of $P_{0}$. Note that $t_{22}^{*}$ in (45) monotonically decreases with $P_{0}$, thus a lower bound of $P_{0}$, denoted as $P_{0}^{L}$, can be obtained by solving the equation $t_{22}^{*}\left(P_{0}\right)=T$ with $L_{2}^{*}=I_{2}$. Based on this $P_{0}^{L}$, we can further obtain a proper upper bound of $P_{0}$, denoted as $P_{0}^{U}$, which should make (P4) feasible and lead to positive energy savings for both of the users, and the optimal $P_{0}^{\star}$ must be in the range of $\left(P_{0}^{L}, P_{0}^{U}\right)$. With the contradiction method, the following lemma showing a property of $P_{0}^{\star}$ can be easily obtained.

Lemma 4. When the minimum feasible $P_{0}^{\star}$ is used in problem (P4), at least one of the two users should use up all its harvested energy, i.e., $E_{\mathrm{s}, 1}^{*}\left(P_{0}^{\star}\right)=0$ or $E_{\mathrm{s}, 2}^{*}\left(P_{0}^{\star}\right)=0$.

\section{Simulation Results}

In this section, the performance of the proposed scheme (UC-JOPT) is investigated by simulations. Also, we include the results of two baselines: UC-ET and IUC, which represent the schemes with equal time allocation for offloading $D_{1}$ 's input data, i.e., $t_{21}=t_{1}=L_{1}^{*} / r_{1,12}\left(\mathbf{p}^{*}\right)$, and with inactive user cooperation by letting $t_{21}=0$ and $t_{1}=\bar{L}_{1}^{*} / r_{1,1}\left(\mathbf{p}^{*}\right)$, respectively. The other variables of these two baselines are obtained from Theorem 1 and Theorem 2. Comparisons with these two baselines can further show the capability of the proposed UC-JOPT scheme in handling CILC tasks and resisting the double-near-far effect in WPCNs.

The simulation parameters are set as follows unless specified otherwise. We set $B=10 \mathrm{MHz}, T=0.2 \mathrm{~s}, N_{0}=10^{-9} \mathrm{~W}$, $\nu_{i}=0.8$ and $\kappa_{i}=10^{-28}$, respectively. It is assumed that the channel reciprocity holds for the downlink and uplink, and thus $g_{1}=h_{1}, g_{2}=h_{2}$. The channel power gain is modeled as $h_{j}=10^{-3} d_{j}^{-\alpha} \phi_{j}, j \in\{1,2,12\}$, where $\phi_{j}$ represents the short-term Rayleigh fading and $\alpha$ is the path-loss exponent. We assume that $d_{1}=10 \mathrm{~m}, d_{2}=6 \mathrm{~m}, d_{12}=6 \mathrm{~m}$ and $\alpha=2$. For each user $D_{i}, i \in\{1,2\}, f_{i}$ is uniformly selected from the set of $\{0.1,0.2, \ldots, 1.0\} \mathrm{GHz}$. Besides, $I_{i}$ and $C_{i}$ follow the uniform distribution with $I_{i} \in[100,500] \mathrm{KB}$ and $C_{i} \in$ $[1000,2000]$ cycles/bit, respectively. The simulated figures in this section are based on 1000 realizations.

Fig. 1 depict the average minimum transmit power (AMTP) versus the same input data size $I=I_{1}=I_{2}$ and the block length $T$ in (a) and (b), respectively. The AMTP of all the schemes increase with $I$ but decrease with $T$, as expected. We can see that the UC-JOPT scheme obviously outperforms the baselines, indicating the significance of the optimization and user cooperation in UC-JOPT. Also, it is noted that the gaps of AMTP between different schemes become more significant for a larger $I$ or a shorter $T$, demonstrating superiority of the proposed UC-JOPT scheme in handling the CILC tasks.

Fig. 2 shows the AMTP with respect to $\xi$ for $\alpha=2,2.5$ where $d_{2}=\xi d_{1}, d_{12}=(1-\xi) d_{1}$, and $\phi_{1}=\phi_{2}$. We can see that the proposed UC-JOPT scheme is superior to the benchmarks, and the improvements are even more pronounced with a larger $\alpha$, indicating that UC-JOPT is highly effective in resisting the attenuation caused by path loss. It is also noticed that the curves of UC-JOPT and UC-ET, first decrease then increase with $\xi$, achieving the minimum AMTP at a saddle point of $\xi$. This is because for the cooperative computation offloading schemes, the performance depends not only on $h_{2}$ but also on $h_{12}$, and there exists a tradeoff between the two values. It is interesting to note that the performance of UCJOPT converges to IUC as $\xi$ gradually tends to 1 since both $D_{1}$ and $D_{2}$ suffer from severe signal attenuation. However, the performance of UC-ET is even worse than IUC when $\xi$ becomes larger approaching to 1 , which shows the importance and effect of optimizing the offloading time fraction.

\section{CONCLUSions}

In this paper, we investigated the use of cooperative communications in computation offloading for a WPT-MEC system. Joint power and time allocation for cooperative computation offloading has been considered with the aim to minimize the transmit power of the AP for completing the computation tasks of the two near-far users. A two-phase method was proposed to find the optimal solution. Simulation results revealed that the proposed scheme greatly outperforms the baselines in handling CILC tasks and resisting double-near-far effect in WPCNs.

\section{ACKNOWLEDGMENT}

This work is supported in part by the U.K. EPSRC under Grants EP/K015893/1 and EP/L026031/1, and in part by the NSFC under Grants 61620106011 and U1705263. 


\section{APPENDIX A}

1) The following lemma is useful to prove the first result, which can be easily proved using the property of derivative.

Lemma 5. For function $q(z)=e^{(m-1) z}-e m z=0(m>0$ is a constant), there exists a unique root on $z \in\left(0, \frac{1}{m}\right)$, which can be easily obtained by a bisection search method.

We will first consider the cases of $M_{1}^{+}>0$ or $\mu_{1} \geq\left(\beta_{1}+\right.$ $\left.\beta_{2}\right) P_{0} / z^{*}$. From (34) and (38), we can get the equation given below

$$
W_{0}\left(-e^{-\left(\frac{\eta^{*} \ln 2}{\lambda_{3}^{*} B}+1\right)}\right)=\frac{-\eta^{*} \ln 2}{\lambda_{3}^{*} B\left(\beta_{1}+\beta_{2}\right) P_{0}} .
$$

Denoting $z^{*}=\frac{\eta^{*} \ln 2}{\lambda_{3}^{*} B}>0$ and using the definition of the Lambert function, the above equation can be rewritten as

$$
e^{\left(\frac{1}{\left(\beta_{1}+\beta_{2}\right) P_{0}}-1\right) z^{*}}-\frac{e}{\left(\beta_{1}+\beta_{2}\right) P_{0}} z^{*}=0
$$

Note that $\beta_{1} \frac{q_{1}^{*}}{t^{*}}=\frac{\lambda_{3}^{*} B\left(\beta_{1}+\beta_{2}\right)}{\eta^{*} \ln 2}-\frac{1}{P_{0}}=\frac{\left(\beta_{1}+\beta_{2}\right)}{z^{*}}-\frac{1}{P_{0}}>0$, which means that equation (49) should have a unique root $z^{*}$ on $\left(0,\left(\beta_{1}+\beta_{2}\right) P_{0}\right)$. According to Lemma 5, solving (49) is equivalent to find the unique root of $q(z)=0$ on $z \in\left(0,\left(\beta_{1}+\right.\right.$ $\left.\left.\beta_{2}\right) P_{0}\right)$ with $m=1 /\left(\beta_{1}+\beta_{2}\right) P_{0}$, and this unique root can be obtained by a bi-section search on $z^{*} \in\left(0,\left(\beta_{1}+\beta_{2}\right) P_{0}\right)$. Therefore, $\lambda_{3}^{*}$ can be expressed as $\lambda_{3}^{*}=\frac{\eta^{*} \ln 2}{B z^{*}}$. Substituting $\lambda_{3}^{*}$ and $\left(1+\lambda_{1}^{*}\right)$ (in (37)), and $\beta_{1}$ into (27) leads to

$$
\frac{\partial \mathcal{L}}{\partial L_{1}^{*}}=\frac{\ln 2}{B}\left(\frac{\mu_{1}}{\left(\beta_{1}+\beta_{2}\right) P_{0}}-\frac{1}{z^{*}}\right) \eta^{*},
$$

which establishes the result of $L_{1}^{*}$ in (39). Similarly, substituting $\lambda_{3}^{*}=\frac{\eta^{*} \ln 2}{B z^{*}}$ into (38), we have

$$
\left(\frac{q_{1}^{*}}{t_{1}^{*}}, \frac{q_{21}^{*}}{t_{21}^{*}}\right)=\left(\frac{1}{\beta_{1}} V\left(z^{*}\right), \frac{1}{\beta_{2}} V\left(z^{*}\right)\right) \succ \mathbf{0},
$$

where $V\left(z^{*}\right)=\frac{\beta_{1}+\beta_{2}}{z^{*}}-\frac{1}{P_{0}}$. Based on these, we can further obtain $p_{1}^{*}$ and $p_{21}^{*}$ through the variable revivification, i.e., $p_{1}^{*}=$ $\nu_{1} g_{1} P_{0} \frac{q_{1}^{*}}{t_{1}^{*}}$ and $p_{21}^{*}=\nu_{2} g_{2} P_{0} \frac{q_{21}^{*}}{t_{21}^{*}}$, leading to the results in (40) and (41). If $M_{1}^{+}=0, \mu_{1}<\left(\beta_{1}+\beta_{2}\right) P_{0} / z^{*}$, it can be derived that $L_{1}^{*}=0$ according to (27), and thus $p_{1}^{*}=0, p_{21}^{*}=0$.

2) Next, we will prove the second result of Theorem 1. Similarly, we also first consider the cases of $M_{2}^{+}>0$ or $\rho\left(\mu_{2}\right) \geq\left(\beta_{1}+\beta_{2}\right) P_{0}$. According to Lemma 3, the optimal transmission rate for offloading $D_{2}$ 's input data, i.e., $\frac{L_{2}^{*}}{t_{22}^{*}}$ can be obtained through (24) as

$$
r_{2}^{*}=\frac{B}{\ln 2}\left[W_{0}\left(\frac{\left(\beta_{1}+\beta_{2}\right) P_{0}-1}{e}\right)+1\right]>0,
$$

where the property of $\lambda_{2}^{*}$ in (37) and the definition of $\beta_{2}$ are used. It is known that $g^{\prime}(x)=\frac{N_{0} \ln 2}{B} 2^{\frac{x}{B}}$ is a monotonically increasing function of $x$. Through (28), we can derive that $\frac{\partial \mathcal{L}}{\partial L_{2}^{*}}(<,=,>) 0$ hold if and only if $\frac{L_{2}^{*}}{t_{22}^{*}}(>,=,<) \frac{B}{\ln 2} \ln \mu_{2}$, respectively. Hence, the result of $L_{2}^{*}$ in (42) can be obtained by comparing the expression of $\frac{L_{2}^{*}}{t_{22}^{*}}$ in $(52)$ and $\frac{B}{\ln 2} \ln \mu_{2}$. Hence, the optimal transmit power for offloading $D_{2}$ 's data is $p_{22}^{*}=\frac{1}{h_{2}} g\left(\frac{L_{2}^{*}}{t_{22}^{*}}\right)$, giving the result in (43). For the case of $M_{2}^{+}=0, \rho\left(\mu_{2}\right)<\left(\beta_{1}+\beta_{2}\right) P_{0}$, it can be derived that $L_{2}^{*}=0$ according to (28), and thus $p_{22}^{*}=0$.

\section{APPENDIX B}

Based on the results of Theorem 1, we can easily derive $t_{22}^{*}$ through $t_{22}^{*}=\frac{L_{2}^{*}}{r_{2}^{*}}$ with the expression of $r_{2}^{*}$ in (52). For the case of $L_{1}^{*}=0$, we understand that $t_{1}^{*}=0$ and $t_{21}^{*}=0$, and thus $t_{0}^{*}=T-t_{22}^{*}$. For the case of $L_{1}^{*}>0$, combining the results of Lemma 2, Lemma 3, and the active time-sharing constraint in (16f), establishes the following equation

$$
t_{1}^{*}+t_{21}^{*}=L_{1}^{*} / r_{1,1}\left(\mathbf{p}^{*}\right)=T-t_{22}^{*}-t_{0}^{*},
$$

which leads to (46). As for the derivation of $\left(t_{1}^{*}, t_{21}^{*}\right)$ when $L_{1}^{*}>0$, we resort to the results of Lemma 2 and Theorem 1 , and further derive the following lemma, which can be proved by contradiction and here we omit the proof to save space.

Lemma 6. The optimal time allocation $\left(t_{1}^{*}, t_{21}^{*}\right)$ for cooperatively offloading $D_{1}$ 's input data satisfies

$$
L_{1}^{*}=L_{1,1}\left(t_{1}^{*}\right)+L_{1,2}\left(t_{21}^{*}\right)=L_{1,12}\left(t_{1}^{*}\right) .
$$

The above lemma is equivalent to that $L_{1}^{*}=\left(t_{1}^{*}+\right.$ $\left.t_{21}^{*}\right) r_{1,1}\left(\mathbf{p}^{*}\right)=t_{1}^{*} r_{1,12}\left(\mathbf{p}^{*}\right)$, from which we can deduce the optimal time division parameters $\left(t_{1}^{*}, t_{21}^{*}\right)$ as in (47).

\section{REFERENCES}

[1] N. Vastardis, and K. Yang, "Mobile social networks: Architectures, social properties, and key research challenges," IEEE Commun. Surv. Tutor, vol. 15, no. 3, pp. 1355-1371, Third Quarter 2013.

[2] K. Yang, Q. Yu, S. Leng, B. Fan, and F. Wu, "Data and energy integrated communication networks for wireless big data," IEEE Access, vol. 4, pp. 713-723, Feb. 2016.

[3] Y. Mao, C. You, J. Zhang, K. Huang, and K. B. Letaief, "Mobile edge computing: Survey and research outlook," IEEE Commun. Surv. Tutor. vol. PP, no. 99, pp. 1-1, Aug. 2017.

[4] C. You, K. Huang and H. Chae, "Energy efficient mobile cloud computing powered by wireless energy transfer," IEEE J. Sel. Areas Commun., vol. 34, no. 5, pp. 1757-1771, May 2016.

[5] Y. Mao, J. Zhang and K. B. Letaief, "Dynamic computation offloading for mobile-edge computing with energy harvesting devices," IEEE $J$. Sel. Areas Commun., vol. 34, no. 12, pp. 3590-3605, Dec. 2016.

[6] R. Kaewpuang, D. Niyato, P. Wang, and E. Hossain, "A framework for cooperative resource management in mobile cloud computing," IEEE $J$. Sel. Areas Commun., vol. 31, no. 12, pp. 2685-2700, Dec. 2013.

[7] C. You, K. Huang, H. Chae, and B. H. Kim, "Energy-efficient resource allocation for mobile-edge computation offloading," IEEE Trans. Wireless Commun., vol. 16, no. 3, pp. 1397-1411, Mar. 2017.

[8] F. Wang, J. Xu, X. Wang, and S. Cui, "Joint offloading and computing optimization in wireless powered mobile-edge computing systems," IEEE Trans. Wireless Commun., vol. PP, no. 99, pp. 1-1, 2018.

[9] H. Ju and R. Zhang, "User cooperation in wireless powered communication networks," proc. IEEE Global Communications Conference (GLOBECOM), Austin, TX, 2014, pp. 1430-1435.

[10] J. N. Laneman, D. Tse, and G. W. Wornell, "Cooperative diversity in wireless networks: Efficient protocols and outage behavior," IEEE Trans. Inform. Theory, vol. 50, no. 11, pp. 3062-3080, Nov. 2004.

[11] Y. Liang and V. V. Veeravalli, "Gaussian orthogonal relay channels: Optimal resource allocation and capacity," IEEE Trans. Inform. Theory, vol. 51, no. 9, pp. 3284-3289, Sep. 2005.

[12] X. Cao, F. Wang, J. Xu, R. Zhang, and S. Cui, "Joint computation and communication cooperation for mobile edge computing," [Online] Available: https://arxiv.org/pdf/1704.06777.pdf

[13] X. Hu, K. K. Wong, and K. Yang "Wireless powered cooperationassisted mobile edge computing," IEEE Trans. Wireless Commun., vol. PP, no. 99, pp. 1-1, 2018.

[14] S. Boyd and L. Vandenberghe, Convex Optimization. Cambridge, U.K.: Cambridge Univ. Press, 2004.

[15] R. Corless, G. Gonnet, D. Hare, D. Jeffrey, and D. Knuth, "On the Lambert W function," Adv. Comput. Math., vol. 5, pp. 329-359, 1996. 